\title{
BMJ Open A systematic review protocol examining the effect of vitamin D supplementation on endothelial function
}

\author{
A Alyami, ${ }^{1}$ MJ Soares, ${ }^{1} \mathrm{JL}$ Sherriff, ${ }^{1} \mathrm{Y}$ Zhao, ${ }^{2} \mathrm{~J} \mathrm{Hallett,}{ }^{3}$ F Coombes ${ }^{4}$
}

To cite: Alyami A, Soares MJ, Sherriff JL, et al. A systematic review protocol examining the effect of vitamin $\mathrm{D}$ supplementation on endothelial function. BMJ Open 2015;5:e006835 doi:10.1136/bmjopen-2014006835

- Prepublication history for this paper is available online To view these files please visit the journal online (http://dx.doi.org/10.1136/ bmjopen-2014-006835).

Received 6 October 2014 Revised 1 May 2015 Accepted 7 May 2015

\section{(1) crossant}

${ }^{1}$ Directorate of Nutrition, Dietetics \& Food Technology, School of Public Health, Curtin Health Innovation Research Institute of Ageing \& Chronic Disease, Curtin University, Perth, Western Australia, Australia

${ }^{2}$ Directorate of Epidemiology \& Biostatistics, School of Public Health, Curtin University, Perth, Western Australia, Australia ${ }^{3}$ Collaboration for Evidence, Research and Impact in Public Health, School of Public Health, Curtin University, Perth, Western Australia, Australia ${ }^{4}$ University Health Service, Support Services, Curtin University, Perth, Western Australia, Australia

Correspondence to Dr MJ Soares:

m.soares@curtin.edu.au

\section{ABSTRACT}

Introduction: Vitamin D has potential benefits for extraskeletal health. These could include an antiinflammatory effect as well as a reduction in endothelial dysfunction. We aim to provide quality evidence for the hypothesis that supplementation with vitamin D will improve endothelial function (EF), possibly through the abrogation of systemic inflammation.

Methods and analysis: We will conduct a systematic review of all randomised controlled trials on vitamin $D$ supplementation and EF lasting 12 weeks or more. The search will cover the period 2000-2015 and include studies that describe direct measures of EF, markers of endothelial cell (EC) activation and if concurrently reported, indicators of systemic inflammation. Study selection will follow the Preferred Reporting Items for Systematic Reviews and Meta-Analyses (PRISMA) guidelines and study quality will be assessed by the Jadad score in addition to an evaluation of allocation concealment and data analysis. If sufficient data are available, a meta-analysis will be conducted. The effect sizes will be generated using Hedges' g score, for both fixed and random effect models. I $^{2}$ statistics and Galbraith plots will be used to assess heterogeneity and identify their potential sources. Potential publication and small sample size bias will be assessed by visual inspections of funnel plots and also Egger's test. Meta-regression analysis (if feasible) will be conducted with restricted maximum likelihood (REML) estimation method, controlling for potential confounders (demographics, study methods, location, etc). A backward elimination process will be applied in the regression modelling procedure. Subgroup analysis, conditional on number of studies retrieved and their sample size, will be stratified on participant disease category, total dose administered, degree of $25(\mathrm{OH}) \mathrm{D}$ change and type of supplement used.

Ethics and dissemination: Formal ethical approval is not required as primary data will not be collected. The results will be disseminated through a peer-reviewed publication, conference presentation and the popular press.

Trial registration number: International Prospective Register for Systematic Reviews (PROSPERO) number CRD42014013523.

\section{INTRODUCTION}

The vascular endothelium plays a pivotal role in the detection and response to

\section{Strengths and limitations of this study}

- Systematic review and meta-analysis of randomised controlled trials.

- Will offer highest level of evidence for informed decisions.

- Potential clarification that the effect of vitamin $\mathrm{D}$ status on endothelial dysfunction is through inflammation.

- Availability of quality studies with direct measures of endothelial function based on the same technique.

- The many inflammatory and endothelial activation biomarkers in the literature hamper collation of outcomes.

- Key words: endothelial function, vitamin D, inflammation, systematic review, randomised controlled trials.

blood-borne signals and changes in haemodynamic forces. Endothelial dysfunction is strongly linked to cardiovascular disease (CVD) ${ }^{1}$ and can predict the occurrence of type 2 diabetes (T2DM). ${ }^{2}$ A chronic lowgrade inflammation is common to many metabolic disorders, ${ }^{3}$ and also underscores endothelial dysfunction. ${ }^{4}{ }^{5}$ Vitamin $\mathrm{D}$ inadequacy is now a global issue and normalisation of status has a potential protective role in conditions such as obesity, CVD and T2DM. ${ }^{6-11}$ Vitamin $\mathrm{D}$ status is related to inflammation. ${ }^{12}{ }^{13}$ The conversion of 25 $(\mathrm{OH}) \mathrm{D}$ to its active form $1,25(\mathrm{OH}) 2 \mathrm{D}$ occurs in immune system cells such as dendritic cells, macrophages, T cells and B cells. ${ }^{14}$ The outcome of $1,25(\mathrm{OH}) 2 \mathrm{D}$ action in these cells is a decreased production of interferon $\gamma$ (INF- $\gamma$ ), interleukin-12 (IL-12), interleukin-6 (IL-6), and interleukin-23(IL-23) with an enhanced production of IL-4. ${ }^{14}$ Vitamin D may also benefit endothelial function (EF). ${ }^{15}{ }^{16}$ The endothelium can convert 25 $(\mathrm{OH}) \mathrm{D}$ to its active form through a specific endothelial $1 \alpha$-hydroxylase. ${ }^{17}$ Interestingly, greater enzyme activity is stimulated by inflammatory cytokines. ${ }^{18}$ Protective effects of vitamin D may then be realised through 
increased nitric oxide (NO) production, decreased oxidative stress, reduced IL-6, vascular cell adhesion molecules (VCAM) and intracellular adhesion molecule (ICAM) among other effects. ${ }^{16}$ Thus the overall impact of adequate vitamin $\mathrm{D}$ in this context would be to decrease both systemic inflammation and endothelial dysfunction.

One of the most important actions of endothelium is the production of $\mathrm{NO}$, which plays a major role in regulating the vessel diameter and, hence resistance, throughout the arterial bed. ${ }^{19}$ However, two other endothelial cell (EC) products, endothelium-derived hyperpolarising factor and prostacyclin (PGI2), also produce vasodilation of the underlying smooth muscle cells, and overall these actions are countered by the vasoconstrictor EC factor endothelin-1 in the regulation of vascular tone. ${ }^{15}$ Endothelial dysfunction is characterised by reduced NO-dependent vascular activity which leads to dysregulation of arterial tone. ${ }^{20}$ However, there is an endothelium independent pathway as well that is determined by the activity of the smooth muscle layer. Sublingual glyceryl trinitrate (GTN) can be used to uncover the influence of this pathway, ${ }^{21}$ as GTN decreases smooth muscle tone leading to vasodilation. ${ }^{21} \mathrm{~A}$ comprehensive assessment of EF usually encompasses the testing of both pathways.

$\mathrm{EF}$ is assessed by testing the vascular reactivity of either coronary or peripheral arteries. Initially the invasive technique of artery catheterisation was used; this assesses endothelial-dependent vasodilation. Subsequently, non-invasive techniques like flow-mediated dilation (FMD) were developed. This is the current gold standard for measuring EF in peripheral arteries. FMD uses ultrasound imaging to detect the endothelial response to shear stress, acetylcholine infusion, and salbutamol inhalation for the assessment of the endothelial-dependent pathway, or sublingual GTN for the endothelial-independent pathway. More recently, pulse contour analysis (PCA), based on a photoplethysmographic recording of the digital volume pulse, has been used to assess $\mathrm{EF}^{22}$ The derived variables, stiffness index and reflective index (RI) reflect large artery stiffness and small artery vascular tone, respectively. ${ }^{23}$ Studies that employ both salbutamol and GTN in conjunction with PCA have been used to report endothelial dysfunction. On the other hand, arterial applanation tonometry uses a sensitive probe applied in turn to the carotid and femoral arteries to detect characteristics of the transmitted waveform. A derived variable is the augmentation index (AIx), the ratio of the pulse pressure at the second systolic peak to the pulse pressure of the first systolic peak. ${ }^{24}$ Other studies have also employed markers of EC activation, like higher plasma levels of soluble VCAM, ICAM, P-selectin and E-selectin as indicators of endothelial dysfunction. ${ }^{15}$ Systemic inflammation is usually measured by the levels of circulating inflammatory biomarkers such as high sensitivity $\mathrm{C}$ reactive protein (CRP), white cell count (WCC), INF- $\gamma$, IL-12, IL-6, IL-23 and IL-4. ${ }^{14}{ }^{25}$ CRP is an interesting marker since its effect on EC activation ${ }^{5}$ could underscore its high prediction of CVD. ${ }^{5}{ }^{25}$ Moreover, strong relationships between WCC, ICAM and fibrinogen and the prediction of CVD has also been documented. ${ }^{126}$

Collectively, there is sufficient evidence to hypothesise that adequate vitamin $\mathrm{D}$ status may directly attenuate endothelial dysfunction (as supported from functional measures and/or markers of EC activation), or act indirectly through the abrogation of systemic inflammation. Two limited narrative reviews on randomised controlled trials (RCTs) did not, however, uncover consistent support for an effect of vitamin D on improvements in EF or decreases in markers of EC activation. ${ }^{15}{ }^{16}$ Clearly there is a need to expand the scope of such findings to arrive at an evidence-based conclusion. To our knowledge there is no published systematic review that addresses our question. Previous systematic reviews in related fields have examined the links between vitamin $\mathrm{D}$ and CRP, ${ }^{27}$ and vitamin $\mathrm{D}$ and blood pressure, ${ }^{28}$ while a narrative review reported on vitamin $\mathrm{D}$, blood pressure, endothelial and renal function of postmenopausal women. ${ }^{29}$ The present systematic review protocol will evaluate potential causal interrelationships between vitamin $\mathrm{D}$ status and $\mathrm{EF}$, and determine whether systemic inflammation is a moderator of the effect. We address our objectives through a comprehensive protocol targeting all RCTs in this area, from 2000 to 2015, in order to confirm or negate this extraskeletal role for vitamin D.

\section{METHODS AND DESIGN \\ Population}

The systematic review will include high quality RCTs on adults aged $>20$ years who have been supplemented with cholecalciferol or calcitriol, and have had measures of EF, EC activation and/or systemic inflammation before and after the interventions. The study population will be restricted to healthy subjects, and overweight or obese who suffer from glucose intolerance, CVD, metabolic syndrome (MetS) or T2DM.

\section{Study design}

This systematic review will consider only randomised controlled trials of good quality.

\section{Search strategy}

The search strategy aims to find published articles only, and will include a three-stage protocol (figure 1). An initial limited search of Medline and Scopus will be undertaken; this will be followed by analysis of the text words contained in the titles and abstracts, and of the index terms used to describe each article. A second search, using all identified keywords and index terms, will then be undertaken across all included databases. In the third step, the reference lists of key articles will be searched for additional studies. Studies will be restricted to the English language and to those published from 2000 to 2015, inclusive. The databases that will be 
1. An initial limited search of Medline and Scopus will be conducted. Search terms such as vitamin D, endothelial function, inflammation, human and RCT will be used.

2. Analysis of the text words in each retrieved article will be used to build a key word list.

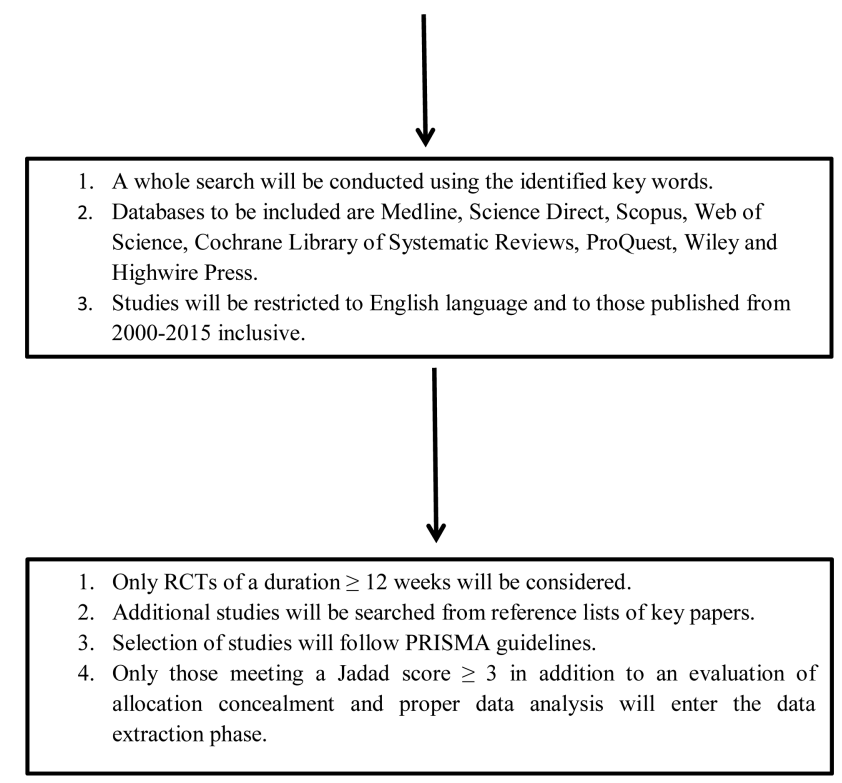

Figure 1 A schematic of the proceses of the systemic review. (Randomised controlled trials (RCTs); Preferred Reporting Items for Systematic Reviews and Meta-Analyses (PRISMA)).

searched are Medline, Science Direct, Scopus, Web of Science, Cochrane Library of Systematic Reviews, ProQuest, Wiley and Highwire Press.

\section{Study selection}

Quantitative studies will be independently assessed by three reviewers and reported using the PRISMA (Preferred Reporting Items for Systematic Reviews and Meta-Analyses) flow diagram. ${ }^{30}$ Valid studies will then be assessed for their quality before any retrieval of information. Any disagreements that arise between the reviewers will be resolved through discussion.

\section{Quality assessment}

The three reviewers will independently check each selected article to minimise bias. All selected articles will be judged for their quality based on the Jadad score ${ }^{31}$ in addition to an evaluation of allocation concealment and data analysis. ${ }^{32}$

\section{Data extraction}

Quantitative data will be extracted from papers receiving a Jadad score of 3 and over with adequate allocation concealment and proper data analysis. ${ }^{31} 32$ The data extracted will include all details specific to the review question and fulfils the requirements for both the narrative synthesis of outcomes and the potential meta-analysis. We will also contact corresponding authors for key information when data are ambiguous or missing from the published study. Data extraction will be independently cross-checked.

\section{Outcomes}

The outcomes of the review will be grouped under the following headings

- EF: this will include direct measures as measured by flow mediated dilation (FMD), PCA, AIx or endothelial vasodilation/vasoconstriction following drug intervention.

- EC activation: these include circulating markers such as P-selectin, E-selectin, L-selectin, VCAM-1, ICAM-1 or von Willebrand factor.

- Systemic inflammatory molecules: these will include markers, such as nuclear factor $\kappa$-light-chainenhancer of activated B cells (NF-KB), proinflammatory cytokine IL-6, IL-12, and high-sensitivity CRP, as well as anti-inflammatory cytokines such as IL-6, IL-10, and adiponectin.

\section{ANALYSIS \\ Descriptive analysis}

A narrative synthesis of the outcomes of the selected studies will be presented in the final review. This will include the following:

1. Type of intervention and the control group and sample size;

2. 25(OH)D3 baseline and final measurement and other biomarkers of interest;

3. Targeted population and its characteristics; age, sex, ethnicity, disease prevalence in group, location and the distance from equator, if possible;

4. Intervention outcomes: this will include the change in $25(\mathrm{OH}) \mathrm{D}$, measurements of EF such as FMD, AIx and PCA derived end points RI and stiffness index, systemic inflammatory biomarkers, EC activation biomarkers, time between last dose and EF measurement.

\section{Statistical analysis}

We are interested in the relationship between vitamin D supplementation and endothelial dysfunction. Endothelial dysfunction is measured in different ways among studies; therefore, we anticipate a limited ability to run a meta-analysis for this review. However, in studies which used the same end point measurements we will report preintervention, postintervention and overall mean change pertaining to the endothelial dysfunction outcomes of interest. The overall mean change will be calculated by subtracting the mean change in the placebo group from that in the treatment group in the studies if these had a parallel design. Standard deviation (SD) will be calculated from standard errors (SEs), or confidence interval (95\% CI), or t or $\mathrm{F}$ value from raw data, where available, for both the placebo group and the treatment group for each study included.

Meta-analysis (where possible) will be carried out to assess the effect of vitamin D supplementation on 
measures of $\mathrm{EF}$ and systemic/vascular inflammation. Effectiveness of vitamin D supplementation on endothelial dysfunction will be reported as standardised mean difference (SMD) for each individual study and its $95 \%$ CI. A positive SMD will denote a higher (more favourable) value in the vitamin D3 group. The effect sizes will be generated using Hedges' $g$ score and presented using a forest plot for each study to assess the magnitude of the intervention effect on a particular outcome. The overall effect sizes will be estimated using both fixed-effects models and random-effects models. $\mathrm{I}^{2}$ statistics and Galbraith plot will be used to assess for heterogeneity and identify the potential sources of heterogeneity. Subgroup analyses, conditional on number of studies retrieved and their sample size, will be stratified on participant disease category (eg, CVD/MetS/T2DM) or total dose administered (daily dose $\times$ duration) (low-medium-high) or degree of $25(\mathrm{OH}) \mathrm{D}$ change (low-medium-high) or supplement used (calcitriol vs vitamin D3 alone vs calcium + vitamin D3).

Potential publication and small sample size bias will be assessed by visual inspections of funnel plots and also Egger's test. To explore the effect of main factors of interest on predicting SMD, meta-regression analysis will be conducted with restricted maximum likelihood (REML) estimation method, controlling for potential confounders (demographics, study methods, and location). Backward elimination process will be applied in the regression modelling procedure. All of the statistical analysis will be performed by using STATA V.12.0 (StataCorp, College Station, Texas, USA). ${ }^{33}$ A $p$ value $<0.05$ will be considered statistically significant for all analyses.

\section{CONCLUSION}

This systematic review will provide evidence in support or against the hypothesis that vitamin $\mathrm{D}$ has a role in EF. This conclusion will stem from direct measurements of EF and/or EC activation, and indirectly through changes in biomarkers of systemic inflammation. Where sufficient data are available, we will conduct a meta-analysis to confirm the relationship between the improvement in vitamin $\mathrm{D}$ status and the reduction in endothelial dysfunction. Moreover, whether this occurs through a reduction in systemic inflammation will also be clarified. Overall, the review will complement the evidence base on the extraskeletal benefits of vitamin $\mathrm{D}$.

Acknowledgements The authors thank the reviewers of the manuscript for their constructive feedback.

Contributors AA, MJS, JLS and JH conceived the idea, planned and designed the study protocol. AA designed the figure and wrote the first draft; $Y Z$ planned the data extraction and statistical analysis; FC provided critical insights. All authors have approved and contributed to the final written manuscript.

Funding This study was supported by intramural funds from School of Public Health, Curtin University. AA is recipient of a PhD scholarship from the Saudi Ministry of Health.
Competing interests None declared.

Provenance and peer review Not commissioned; externally peer reviewed.

Open Access This is an Open Access article distributed in accordance with the Creative Commons Attribution Non Commercial (CC BY-NC 4.0) license, which permits others to distribute, remix, adapt, build upon this work noncommercially, and license their derivative works on different terms, provided the original work is properly cited and the use is non-commercial. See: http:// creativecommons.org/licenses/by-nc/4.0/

\section{REFERENCES}

1. Pearson TA, Mensah GA, Alexander RW, et al. Markers of inflammation and cardiovascular disease application to clinical and public health practice: a statement for healthcare professionals from the centers for disease control and prevention and the American Heart Association. Circulation 2003;107:499-511.

2. Meigs JB, Hu FB, Rifai N, et al. Biomarkers of endothelial dysfunction and risk of type 2 diabetes mellitus. JAMA 2004;291:1978-86.

3. Hotamisligil GS. Inflammation and metabolic disorders. Nature 2006;444:860-7.

4. Huang AL, Vita JA. Effects of systemic inflammation on endotheliumdependent vasodilation. Trends Cardiovasc Med 2006;16:15-20.

5. Szmitko PE, Wang $\mathrm{CH}$, Weisel RD, et al. New markers of inflammation and endothelial cell activation part I. Circulation 2003;108:1917-23.

6. Holick MF, Chen TC. Vitamin D deficiency: a worldwide problem with health consequences. Amer J Clin Nutr 2008;87:1080S-6S.

7. Peterlik M, Cross H. Vitamin D and calcium insufficiency-related chronic diseases: molecular and cellular pathophysiology. Euro $J$ Clin Nutr 2009;63:1377-86.

8. Heaney RP. Vitamin D in health and disease. Clin J Am Soc Nephrol 2008;3:1535-41.

9. Hossein-nezhad A, Holick MF. Vitamin D for health: a global perspective. Mayo Clin Proc 2013;88:720-55.

10. Mitri J, Muraru M, Pittas A. Vitamin D and type 2 diabetes: a systematic review. Eur J Clin Nutr 2011;65:1005-15.

11. Soares MJ, Pathak K, Calton EK. Calcium and vitamin d in the regulation of energy balance: where do we stand? Int J Mol Sci 2014;15:4938-45.

12. Lai YH, Fang TC. The pleiotropic effect of Vitamin D. ISRN Nephrol 2013;2013:898125

13. Calton $\mathrm{E}$, Keane $\mathrm{K}$, Soares $\mathrm{M}$. The potential regulatory role of vitamin D in the bioenergetics of inflammation. Cur Opin Clin Nutr Metab Care 2015;18:367-73.

14. Mora JR, Iwata M, von Andrian UH. Vitamin effects on the immune system: vitamins A and D take centre stage. Nat Rev Immunol 2008;8:685-98.

15. Alyami A, Soares M, Sherriff J, et al. Vitamin D \& endothelial function. Indian J Med Res 2014;140:483-90.

16. Dalan R, Liew H, Tan WKA, et al. Vitamin D and the endothelium: basic, translational and clinical research updates. IJC Metab Endocr 2014;4:4-17.

17. Merke J, Milde P, Lewicka S, et al. Identification and regulation of 1, 25-dihydroxyvitamin D3 receptor activity and biosynthesis of 1, 25-dihydroxyvitamin D3. Studies in cultured bovine aortic endothelial cells and human dermal capillaries. J Clin Invest 1989; 83:1903-15.

18. Zehnder D, Bland R, Chana RS, et al. Synthesis of 1 , 25-dihydroxyvitamin D3 by human endothelial cells is regulated by inflammatory cytokines: a novel autocrine determinant of vascular cell adhesion. J Am Soc Nephrol 2002;13:621-9.

19. Goodfellow J, Owens D, Henderson A. Cardiovascular syndromes $\mathrm{X}$, endothelial dysfunction and insulin resistance. Diabetes Res Clin Pract 1996;31:S163-71

20. Kim JA, Montagnani M, Koh KK, et al. Reciprocal relationships between insulin resistance and endothelial dysfunction: molecular and pathophysiological mechanisms. Circulation 2006;113:1888-904.

21. Yaginuma T, Avolio A, O'Rouke M, et al. Effect of glyceryl trinitrate on peripheral arteries alters left ventricular hydraulic load in man. Cardiovasc Res 1986;20:153-60.

22. Woodman RJ, Watts GF. Measurement and application of arterial stiffness in clinical research: focus on new methodologies and diabetes mellitus. Med Sci Monit 2003;9:RA81-9.

23. Woodman RJ, Watts GF, Kingwell BA, et al. Interpretation of the digital volume pulse; its relationship with large and small artery compliance. Clin Sci 2003;104:283-5. 
24. Barac A, Campia U, Panza JA. Methods for evaluating endothelial function in humans. Hypertension 2007;49:748-60.

25. Ridker PM. Inflammatory biomarkers and risks of myocardial infarction, stroke, diabetes, and total mortality: implications for longevity. Nutr Rev 2007;65(Suppl 3):S253-9.

26. Witkowska AM. Soluble ICAM-1: a marker of vascular inflammation and lifestyle. Cytokine 2005;31:127-34.

27. Chen N, Wan Z, Han SF, et al. Effect of vitamin D supplementation on the level of circulating high-sensitivity C-reactive protein: a metaanalysis of randomized controlled trials. Nutrients 2014;6:2206-16.

28. Beveridge $L A$, Struthers $A D$, Khan F, et al. Effect of vitamin $D$ supplementation on blood pressure: a systematic review and meta-analysis incorporating individual patient data. JAMA Intern Med 2015;175:745-54.
29. Liu ZM, Woo J, Wu SH, et al. The role of vitamin D in blood pressure, endothelial and renal function in postmenopausal women. Nutrients 2013;5:2590-610.

30. Moher D, Liberati A, Tetzlaff $\mathrm{J}$, et al. Preferred reporting items for systematic reviews and meta-analyses: the PRISMA statement. Ann Intern Med 2009;151:264-9.

31. Jadad AR, Moore RA, Carroll D, et al. Assessing the quality of reports of randomized clinical trials: is blinding necessary? Control Clin Trials 1996;17:1-12.

32. Centre for Evidence in Transplantation. Assessment of randomised controlled trials (RCTs). 2010. http://www.transplantevidence.com/ library rct.php (accessed 08-08-2014).

33. Hamilton L. Statistics with STATA: Version 12. USA: Cengage Learning, 2012. 